\title{
Is stoma care effective in terms of morbidity in complicated ileostomies?
}

This article was published in the following Dove Press journal:

International Journal of General Medicine

II August 2015

Number of times this article has been viewed

\section{Pinar Sarkut \\ Halit Ziya Dundar \\ Ismail Tirnova \\ Ersin Ozturk \\ Tuncay Yilmazlar}

Department of General Surgery, Faculty of Medicine, Uludağ University, Görükle, Turkey
Correspondence: Pinar Sarkut

Department of General Surgery, Faculty of Medicine, Uludağ University, Görükle,

16059 Bursa, Turkey

Tel +902242952040

Fax +902244428398

Email pinartasar@gmail.com
Background: Performing transient or permanent ileostomy is one of the common procedures involved in colorectal surgery. Complication rates up to $40 \%$ have been reported in ileostomies. In this report, the effect of specific stoma care unit on ileostomy and its complications were investigated.

Methods: A total of 141 patients, who were operated and underwent ileostomy, due to different causes, at Department of General Surgery, Uludağ University, Bursa, Turkey, between 2003 and 2006, were examined, retrospectively. Patient records were examined in terms of age, sex, surgery indications, urgent/elective state, benign/malign origin, ileostomy type, complications and stoma care, and education. $\chi^{2}$ test was used to compare the categorical data.

Results: Among the patients, 95 (67\%) were male and $46(33 \%)$ were female. The mean age was 47 years (17-67). Some of the subjects (49\%) were operated urgently and some $(51 \%)$ were under elective conditions. The ileostomy types used included the following: end ileostomy (43\%), loop ileostomy (46\%), and double-barrel ileostomy (11\%). Permanent ileostomy was performed in 23 patients and transient ileostomy was performed in 118 patients. The patients were operated because of either benign (48\%) or malign (52\%) causes. Complications developed in $37(26 \%)$ patients. The rate of development of complication was markedly higher in ileostomies performed under urgent conditions $(61 \%$ vs $39 \%)(P<0.001)$. The complications included mucocutaneous separation (12 patients), maceration in the peristomal skin (ten patients), retraction (five patients), necrosis (three patients), prolapsus (three patients), and other metabolic complications (four patients). The complications were treated with care $(68 \%)$ and surgical revision (32\%)

Conclusion: The rate of ileostomy was found to be higher in the male patients compared to female patients. The risk of development of complications was found to be higher in ileostomies performed under urgent conditions. The most common complication observed was mucocutaneous separation. Ileostomy complications can be treated conservatively with professional care and education.

Keywords: ileostomy, stoma care, complications

\section{Introduction}

Transient or permanent ileostomy is a procedure which is frequently performed during interventions of colorectal surgery. It decreases the clinical effects of complications including fistula and leakage, which may develop secondary to distal colorectal and coloanal anastomoses performed especially in patients who received preoperative radiation. However, some complications related with the stoma may develop during the process of opening and closing the stoma. Most of the time, these complications can be treated via conservative approaches. Risk factors for the complications, 
which might develop after ileostomy, vary according to the patient, disease, and surgical technique. Advanced age and comorbidity increase the rate of complications of ileostomy. In obese patients with fatty mesentery and thick subcutaneous tissue, the frequency of complications including retraction is higher. However, stoma care with experienced stoma care nurses decreases the frequency of these complications and improves the quality of the life in patients with complicated ileostomy.

In our study, we evaluated the efficiency of specific stoma care units and professional care on treatment of ileostomy complications.

\section{Materials and methods}

The patients $(\mathrm{N}=141)$, who underwent ileostomy because of different causes in Department of General Surgery, Uludağ University, Bursa, Turkey, between 2003 and 2006, were examined, retrospectively. The data of the patients were evaluated in terms of age, sex, surgery indications, urgent/elective state, malign/benign origin of the pathology, type of ileostomy, complications and stoma care, and education. Pregnant women, individuals who regularly used steroids and who had any autoimmune disorder involving the musculoskeletal system, were excluded from the study. $\chi^{2}$ test was used to evaluate the categorical data.

\section{Results}

Among the patients, 95 (67\%) were male and 46 (33\%) were female. The mean age was 47 years (17-67). In all, 49\% of the subjects were operated urgently and $51 \%$ were operated under elective conditions. There were three types of ileostomy: end ileostomy (43\%), loop ileostomy (46\%), and doublebarrel ileostomy (11\%). Permanent ileostomy was performed in 23 patients and transient ileostomy was performed in 118 patients. The patients were operated because of benign (48\%) and malign (52\%) causes. Complications were developed in 37 (26\%) patients; and the rate of the development was $61 \%$ in ileostomies performed under urgent conditions, while it was 39\% in ileostomies performed under elective conditions $(P<0.001)$. Patients who were operated because of malign causes constituted $67 \%$ of the patients who developed complications $(P=0.09)$. Complications developed in $53 \%$ of the patients with double-barrel ileostomy, in $26 \%$ of the patients who underwent loop ileostomy, and in $19 \%$ of the patients with end ileostomy. The complications are shown in Table 1, among them the most common was mucocutaneous separation. Forty-one percentage of the cases of mucocutaneous separation occurred in the patients who underwent loop ileostomy.
Table I Patients in whom complications were observed after ileostomy

\begin{tabular}{ll}
\hline Complications & $\mathbf{N}$ \\
\hline Mucocutaneous separation & 12 \\
Skin maceration & 10 \\
Retraction & 5 \\
Necrosis & 3 \\
Prolapsus & 3 \\
Metabolic complications & 4 \\
\hline
\end{tabular}

Surgical treatment was performed in $12(32 \%)$ of the patients who developed complications. Sutures were placed in four of them, the place of ostomy was changed in two and ostomy revision was performed in six because of necrosis and prolapsus. Conservative treatment with the professional ostomy care was performed in the remaining 25 (68\%) patients. The mean number of the care given to the patients during the hospitalization was 10.5 and the mean number of the education given to the patients and their relatives was 8.5.

\section{Discussion}

In this study, we observed significantly more complications in patients who underwent ostomy under urgent conditions. These complications were affected by the surgical techniques and factors related with the patients. It can be inferred that preparation of patients in a more appropriate way with a multidisciplinary team would be an effective factor in elective cases. The patients in whom elective ostomy was planned to be performed, the ostomy place was marked in the preoperative period by the ostomy care nurses. These planned cases were evaluated in the preoperative period and marking was done by determining exactly the skin folds and spaces formed by sitting and standing positions. In the cases when ostomies were performed under urgent conditions, the place of ostomy could not be planned before operation. Retractions were observed, especially in fat patients in the postoperative period at places where ostomy coincided with abdominal skinfold. Again, separations in the ostomy bags which required to be adhered to the place of folding, cause the intestinal content to contact with the skin and may lead to severe skin macerations. Therefore, the possibility of skin macerations, mucocutaneous separation, and even retractions, which occur frequently in the postoperative period, was decreased in elective cases.

Complications including necrosis and prolapsus were observed at a rate of $16 \%$ in the patients. Surgery was also involved in the treatment of these complications. Surgical intervention was performed in only six of the patients 
who developed mucocutaneous separation and retraction. The remaining eleven patients were treated with ostomy care in the postoperative period. Skin complications, which are observed most commonly and considerably disrupt the quality of life, were also the most common complications in our study with a rate of $32 \%$. The most important factor involved in development and treatment of such complications is ostomy care. In our study, ostomy complications which developed in the postoperative period were treated with regular ostomy care in a total of 25 patients. This decreases the cost per patient and minimizes surgical trauma. Therefore, education and information given by ostomy nurses during the preoperative and postoperative periods prevent most of these complications and increase the quality of life.

The rates of complications have been reported to range from $6 \%$ to $59 \%$ in the studies which examined the types of stoma complications. ${ }^{1-6}$ It has been proposed that surgical techniques, selection of the stoma place, informing patients and emotional support are the most important factors which are effective in the success of stoma surgery. Retraction, hernia, peristomal skin problems, necrosis, and prolapsus have been reported to be the most common types of complications. ${ }^{7}$

Peristomal skin problems are observed to range from $18 \%$ to $55 \%$ in the patients with ostomy. ${ }^{8}$ Cutaneous complications include problems ranging from mild cutaneous irritation to ulceration. Selection of the wrong stoma place, and complications including prolapse and retraction increase cutaneous complications which are observed frequently. The greatest risk in patients who develop peristomal cutaneous complications is lack of access to wound--ostomy care nurses. ${ }^{9}$ Minor problems related with stoma may cause higher cost and higher hospitalization rates without wound-ostomy care nurses. ${ }^{10}$

Superficial necrosis is observed in $2 \%-20 \%$ of the patients, and deep necrosis is observed in $0.37 \%-3 \% .{ }^{11-14}$ The bowel prepared for ostomy to prevent ischemia should be able to move easily up to the place where it will exit the skin, and the vascularity of the bowel should not be disrupted during this process. Epiploic fat and mesentery should not be damaged too much.

Prolapse is frequently a late complication and observed with a rate of $2 \%-20 \%$. It is most frequently related with loop ostomies. ${ }^{3,15}$ Stomal prolapse disturbs the comfort of the patients. Since the stoma usually maintains its function, urgent surgery is rarely needed.
Potential complications are important both in terms of surgical mortality and the quality of life in the future, although they are observed only in a few patients who had undergone the major operations in whom transient ileostomies were performed. The structure of the body of the patient, abdominal skin scars due to previous history, and abdominal wall thickness are considerably important in the selection and care of the ostomy place. Ostomy places planned and drawn by ostomy nurses, educations related with ostomy care in the postoperative period, and the minimization of surgical trauma by correcting complications conservatively should be main targets of a preventable morbidity.

\section{Disclosure}

The authors report no conflicts of interest in this work.

\section{References}

1. Ratliff CR, Donovan AM. Frequency of peristomal complications. Ostomy Wound Manage. 2001;47:26-29.

2. Duchesne JC, Wang YZ, Weintraub SL, Boyle M, Hunt JP. Stoma complications: a multivariate analysis. Am Surg. 2002;68:961-966.

3. Park JJ, Del Pino A, Orsay CP, et al. Stoma complications: the Cook County Hospital experience. Dis Colon Rectum. 1999;42:1575-1580.

4. Londono-Schimmer EE, Leong AP, Phillips RK. Life table analysis of stomal complications following colostomy. Dis Colon Rectum. 1994;37: 916-920.

5. Chen F, Stuart M. The morbidity of defunctioning stomata. Aust NZJ Surg. 1996;66:218-221.

6. Porter JA, Salvati EP, Rubin RJ, Eisenstat TE. Complications of colostomies. Dis Colon Rectum. 1989;32:299-303.

7. Kwiatt M, Kawata M.Avoidance and management of stomal complications. Clin Colon Rectal Surg. 2013;26:112-121.

8. Colwell JC, Goldberg M, Carmel J. The state of the standard diversion. J Wound Ostomy Continence Nurs. 2001;28:6-17.

9. Marquis P, Marrel A, Jambon B. Quality of life in patients with stomas: the Montreux Study. Ostomy Wound Manage. 2003;49:48-55.

10. Turnbull GB. Stomal complications: at what price? Ostomy Wound Manage. 2003;49:17-18.

11. Formijne Jonkers HA, Draaisma WA, Roskott AM, van Overbeeke AJ, Broeders IA, Consten EC. Early complications after stoma formation: a prospective cohort study in 100 patients with 1-year follow-up. Int $J$ Colorectal Dis. 2012;27:1095-1099.

12. Nastro P, Knowles CH, McGrath A, Heyman B, Porrett TR, Lunniss PJ. Complications of intestinal stomas. Br J Surg. 2010;97:1885-1889.

13. Cottam J, Richards K, Hasted A, Blackman A. Results of a nationwide prospective audit of stoma complications within 3 weeks of surgery. Colorectal Dis. 2007;9:834-838.

14. Parmar KL, Zammit M, Smith A, Kenyon D, Lees NP; Greater Manchester and Cheshire Colorectal Cancer Network. A prospective audit of early stoma complications in colorectal cancer treatment throughout the Greater Manchester and Cheshire colorectal cancer network. Colorectal Dis. 2011;13:935-938.

15. Harris DA, Egbeare D, Jones S, Benjamin H, Woodward A, Foster ME Complications and mortality following stoma formation. Ann $\mathrm{R}$ Coll Surg Engl. 2005;87:427-431. 


\section{Publish your work in this journal}

The International Journal of General Medicine is an international, peer-reviewed open-access journal that focuses on general and internal medicine, pathogenesis, epidemiology, diagnosis, monitoring and treatment protocols. The journal is characterized by the rapid reporting of reviews, original research and clinical studies across all disease areas.

A key focus is the elucidation of disease processes and management protocols resulting in improved outcomes for the patient. The manuscript management system is completely online and includes a very quick and fair peer-review system. Visit http://www.dovepress.com/ testimonials.php to read real quotes from published authors.

Submit your manuscript here: http://www.dovepress.com/international-journal-of-general-medicine-journal 